\title{
The NHS Infection Intelligence Platform
}

\author{
Professor Dilip Nathwani \\ Ward 42, East Block \\ Ninewells Hospital \& Medical School, \\ Dundee \\ DD19SY \\ Dilip.Nathwani@nhs.net
}

Ken Monaghan

NHS National Services Scotland

Meridian Court

5 Cadogan Street

Glasgow

G2 6QE

Ken.Monaghan@nhs.net

\author{
Professor Marion Bennie \\ NHS National Services Scotland \\ Gyle Square \\ 1 South Gyle Crescent \\ Edinburgh EH12 9EB \\ m.bennie@nhs.net
}

Dr Charis Marwick

NHS Tayside/University of Dundee

Division of Population Health Sciences

University of Dundee

DD2 4BF

c.z.marwick@dundee.ac.uk charismarwick@nhs.net

\author{
William Malcolm \\ NHS National Services Scotland \\ Meridian Court \\ 5 Cadogan Street \\ Glasgow \\ G2 6QE \\ W.Malcolm@nhs.net \\ Emese Toth
}

NHS National Services Scotland

Gyle Square

1 South Gyle Crescent

Edinburgh EH12 9EB

Emese.Toth@nhs.net

\begin{abstract}
We describe the NHS Infection Intelligence Platform (IIP) as a comprehensive, dynamic and responsive infection surveillance and research resource for Scotland. The IIP will integrate many different strands of electronic infection data in a safe and secure environment, with the aim of improving public health. It will harness existing health data to support clinicians in improving patient outcomes and reduce harm from community and healthcare acquired infection.
\end{abstract}

Infection, Informatics, Healthcare Improvement, Antimicrobial, Outcomes.

\section{INTRODUCTION}

The NHS Infection Intelligence Platform (IIP) is a comprehensive, dynamic and responsive resource capability for Scotland that will harness existing health data to support clinicians in improving patient outcomes and reduce harm from community and healthcare acquired infection [1].

The IIP programme's vision consists of "improving patient outcomes and reducing harm from infection through innovative data integration, to support clinicians within the NHS in Scotland". The IIP programme is now completing its second year of work. The early focus has been on delivering the necessary governance and infrastructure to integrate key national datasets, including antimicrobial prescribing, antimicrobial resistance and hospital activity datasets. The next 12 months will be focussed on delivering clinical studies to generate a clinical evidence base for policy and practice change in infection prevention and antimicrobial stewardship.

NHSScotland is currently investing in the development of the IIP, funded by the Scottish Government Healthcare Associated Infection (HAI) Task Force in 2013 for a three-year period [2]. The IIP is a central plank of the Scottish Government's recently published ScotMARAP2 (Scottish
Management of Antimicrobial Resistance Action Plan) document, which is focussed on reducing antimicrobial resistance over the next 5 years [3].

\section{INFECTION INTELLIGENCE PLATFORM OVERVIEW}

\subsection{Rationale for an Infection Intelligence Platform}

Community and healthcare associated infections are a significant health burden and are a priority for NHSScotland. NHS National Services Scotland (NSS) [4] currently hosts a wide range of infection related data on behalf of NHSScotland. The NHSScotland Infection Intelligence Platform is being developed to improve patient outcomes and reduce harm from innovative infection data integration to support clinicians across the NHS in Scotland.

\subsection{Scope of the Infection Intelligence Platform}

The IIP will support clinicians by providing integrated information on infection (for example by combining risk factors, demographics, healthcare activity, medicines use and clinical data) through linking together the many strands of national and local infection information collected across 
NHSScotland. NSS has been tasked with the development and implementation of IIP and is supported by a broad coalition of clinicians and national stakeholders.

\subsection{Implication for Clinicians}

The IIP will allow better use of our national and local data through a "collect once and use often" approach which will enable faster and more efficient information to clinicians to improve care, reduce harm and reduce variation across NHSScotland.

\subsection{Implication for NHSScotland}

The creation of the first Infection Intelligence Platform in the UK is an exciting innovation in the prevention and treatment of infection and confirms Scotland's position as a world leader in healthcare informatics.

\subsection{Technical Design}

The data are linked in the NSS Corporate Data Warehouse which uses Kimball design methodology on an Oracle 11.2 platform.

Data visualisation will be provided using Tableau 8.2 whilst ad hoc reporting capability will be provided using Business Objects XI3 (soon to be upgraded to XI4).

\section{FURTHER INFORMATION}

If you wish to receive further information about the development of IIP, please visit the IIP website (www.isdscotland.org/iip) or contact the IIP team (NSS.iip@nhs.net).

\section{REFERENCES}

[1] Infection Intelligence Platform. Information Service Division.

http://www.isdscotland.org/Health-Topics/Healthand-Social-Community-Care/InfectionIntelligence-Platform/

[2] Healthcare Associated Infection Taskforce. Scottish Government. http://www.gov.scot/Topics/Health/Services/Prev enting-Healthcare-Infections/InfectionMonitoring/Taskforce

[3] Scottish Management of Antimicrobial Resistance Action Plan 2014-18 (ScotMARAP2). Published by the Scottish Government, July 2014. 18p. ISBN: 978-1-78412-726-8 http://www.gov.scot/Resource/0045/00456736.p $\underline{\mathrm{df}}$

[4] NHS National Services Scotland. http://www.nhsnss.org/ 